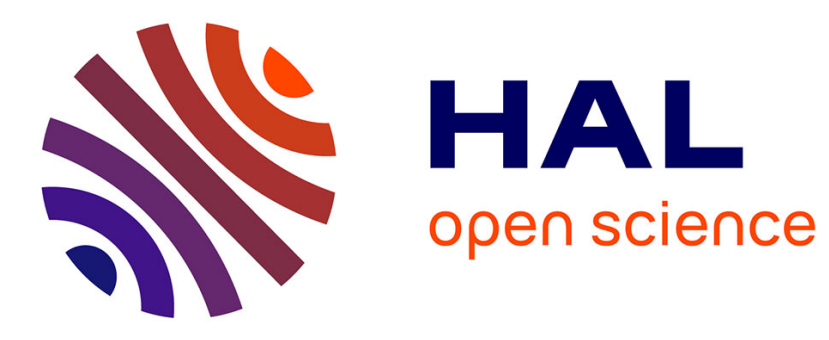

\title{
Vehicle localization from inaccurate telemetric data: a set inversion approach
}

Olivier Leveque, Luc Jaulin, Dominique Meizel, Eric Walter

\section{To cite this version:}

Olivier Leveque, Luc Jaulin, Dominique Meizel, Eric Walter. Vehicle localization from inaccurate telemetric data: a set inversion approach. 5th IFAC Symposium on Robot Control SY.RO.CO.'97, Sep 1997, Nantes, France. pp.179-186. hal-00844041

\section{HAL Id: hal-00844041 https://hal.science/hal-00844041}

Submitted on 12 Jul 2013

HAL is a multi-disciplinary open access archive for the deposit and dissemination of scientific research documents, whether they are published or not. The documents may come from teaching and research institutions in France or abroad, or from public or private research centers.
L'archive ouverte pluridisciplinaire HAL, est destinée au dépôt et à la diffusion de documents scientifiques de niveau recherche, publiés ou non, émanant des établissements d'enseignement et de recherche français ou étrangers, des laboratoires publics ou privés. 


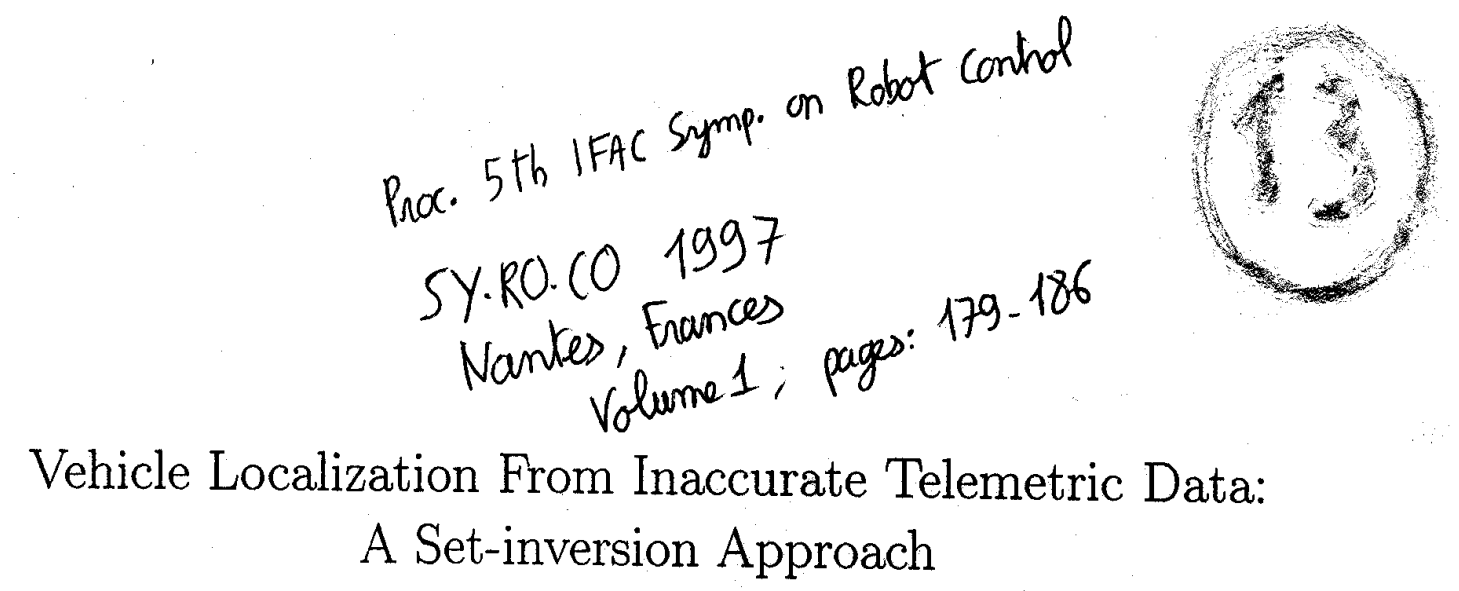

\author{
O. LEVEQUE * , L. JAULIN **, D. MEIZEL *, E. WALTER *** \\ * UTC/HEUDIASYC.URA CNRS 817 \\ B.P. 529, F-60205 COMPIEGNE CEDEX FRANCE \\ e-mail: Olivier.Leveque@utc.fr \\ tel: (33) 03-44-23-44-23 \\ fax: (33) 03-44-23-44-77 \\ ** UNIVERSITE D'ANGERS, FRANCE \\ *** LABORATOIRE DES SIGNAUX ET DES SYSTEMES, CNRS-SUPELEC, FRANCE
}

Keywords: Multi-sensor Fusion, Vehicle Localization, Set-membership Estimation, Set Inversion, Interval Analysis.

\footnotetext{
Abstract

In this communication, vehicle localization in a 2D-mapped environment from inaccurate telemetric measurements is stated as a set-inversion problem. A novel methodology based upon a mechanical interpretation of a range measurement and interval analysis is presented. The approach aims at characterizing the set $\mathcal{Q}$ of all vehicle configurations which are consistent with all range measurements, their associated inaccuracies and the environment model. It provides a global, guaranteed and accurate characterization of the solution set $\mathcal{Q}$, even when this set is non-connected. Moreover, the method naturally produces and manages the multiple hypothesis of feature-measurement association.
}

\section{INTRODUCTION}

As the process of determining the position and orientation of a vehicle with respect to a global reference frame, localization is one of the fundamental skills required for autonomous navigation in a working environment. Due to autonomy restriction which implies that the vehicle must only use its aboard sensors to locate itself, a direct configuration measurement is not available and localization must be obtained by estimation. Hence, different approaches have been proposed to address the difficult question: "where am I?". Many vehicle localization methods reported in the literature use statistical state estimation techniques, like Extended Kalman Filtering, to provide a point estimate associated with a confidence region which quantifies the estimation accuracy. Under the assumptions of small variations and noise statistical modelling, these methods are simple to use and efficient but they require good initial estimates and a good feature-measurement association, [18] [11] [3] [2]. An attractive alternative to such a statistical approach is set-membership estimation [19] [15] [16] [13] which takes advantages of bounds on measurement 
inaccuracy to deduce from observed data a set of possible configurations for the vehicle [1] [5] [4]. This allows a natural representation of both the measurement error and the set of configuration estimations. A $\dot{s}$ in a statistical context, estimation is simpler when the error is affine in the parameters and application of set-membership estimation to vehicle localization has so far relied on a linearization of the error equation, which led to approximate solutions [13]. In this paper, we introduce a new set-membership approach for the problem of vehicle localization in a mapped environment from inaccurate telemetric data. The proposed methodology, based upon a mechanical interpretation of the range measurement, defines localization as a set-inversion problem. Afterwards, an algorithm based on interval analysis is used to solve this set-inversion problem and provides a non necessarily connected set of all vehicle configurations which are consistent with both the telemetric measurements, their associated inaccuracies and the world model. Thus, each connected component of this solution set corresponds to a matching hypothesis. The measurement error is assumed to be unknown but bounded and the simulator of the model equations can be viewed as a function $\mathbf{f}$ which associates to any given vehicle configuration $\mathbf{q}$ the vector of all associated telemetric data. Localization is thus performed by inverting the measurement set generated by $f$. Besides the natural management of multiple hypothesis, this approach produces a set-membership estimate of the vehicle configuration which is global, guaranteed and accurate. In contrast with previous approaches, it does not involve any linearizing approximation.

This paper is organized as follows; the problem and the principle used to solve it will be defined in Section 2. The set-inversion approach for vehicle localization will be detailed in Section 3. Finally, a simulation result will be presented to highlight the performance of this approach.

\section{LOCALIZATION}

\subsection{Problem Statement}

Consider the static localization problem of a singlebody vehicle in a $2 \mathrm{D}$-mapped environment. The vehicle is able to perform displacements on a plane floor and its configuration $q=\left(x_{C}, y_{C}, \theta\right)^{T}$ is defined by the coordinates of a characteristic point $C$ together with the vehicle orientation $\theta$ as illustrated in Figure 1. The configuration space $\mathcal{C}$ is defined by $\mathcal{C}=\Re^{2} \times \mathcal{S}^{1}$ with $\mathcal{S}^{1}=[-\pi,+\pi[\bmod 2 \pi$. Let $\mathcal{M}$ be a reference frame tied to the mobile and let $\mathcal{W}$ be a fixed frame called world-reference frame. By convention, primed variables $\left(x^{\prime}, y^{\prime}, \ldots\right)$ are coordinates defined in the mobile frame $\mathcal{M}$ whereas non-primed ones $(x, y, \ldots)$ are defined in the fixed frame $\mathcal{W}$. The vehicle can

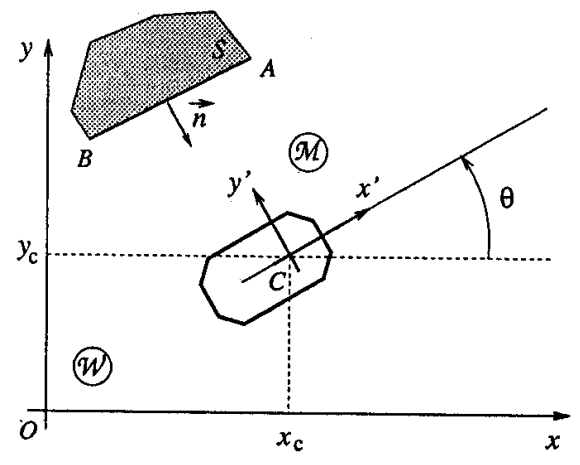

Figure 1: Vehicle configuration and map feature.

feel its environment by using a belt of ultrasonic Polaroid range sensors. The environment is described by a polygonal approximation. Thus the world map consists of a collection of line segments. From a practical viewpoint, the world reference frame is defined by using a subset of the map features. A line segment is commonly defined by its endpoints $A$ and $B$. By convention, its reflecting side is called front side and is defined by the direction of $\vec{n}$ such as $(\overrightarrow{A B}, \vec{n})$ is a direct dihedral. Localizing the vehicle means estimating its actual position and orientation in the fixed reference frame or more specifically the geometrical transformation from the mobile frame to the fixed reference one. 


\subsection{Localization Principle}

For the sake of clarity, the localization principle is first stated in the ideal case when perfect telemeters are used. The inaccuracy of the real ultrasonic sensor will be taken into account later by converting the exact solution obtained in the ideal case into a set of possible solutions which are admissible with respect to measurement inaccuracy. A telemeter (see Figure 2) the position $\left(x_{T}^{\prime}, y_{T}^{\prime}\right)$ and orientation $\alpha_{T}$ of which are known in the mobile frame $\mathcal{M}$, emits a wave that is reflected by a surface element of an environment feature and received back. The time lag between emitted and received signals gives a distance measurement that is the one of the nearest object in the target direction. This distance $d$ and the angle $\alpha_{T}$ being known, this telemetric measurement can be interpreted as a mechanical link between the sensor and the map feature. Localizing the vehicle can then be viewed as fixing it in the environment by means of those virtual mechanical links. Each telemetric measurement is seen as a rigid mechanical component, like a bar. The length of this bar is equal to the measured distance and the bar joins the detected obstacle to the vehicle by means of two different virtual links, (see Figure 2). The first link modelled at the sen-

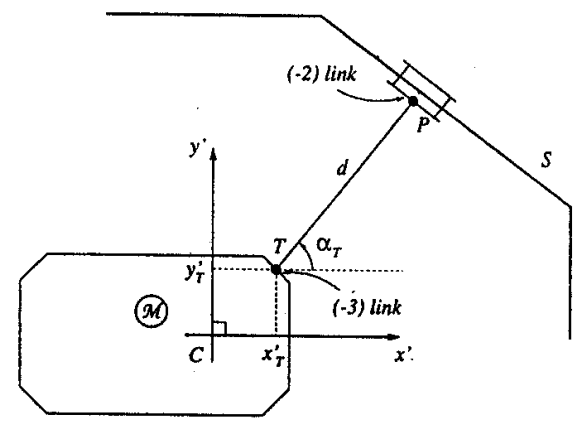

Figure 2: Mechanical interpretation of a range measurement.

sor point $T$ between the sensor (tied to the vehicle) and the emitted ray, is rigid. The second link defined between the detected point $P$ and the map feature $S$ removes one degree of freedom $(P \in S)$. Thus, because of the previous rigid link, one measurement removes one degree of freedom to the vehicle configuration. The fact that three distance measurements from three vehicle sensors to at least two non parallel map features are sufficient to localize the vehicle is an intuitive mechanism theory result. In such an ideal case the configuration is unique. We propose now to take into account the sensor inaccuracy which produces a dispersion of the possible configurations. This dispersion will be expressed by the solution set $\mathcal{Q}$ which contains all the configuration vectors consistent with the telemetric measurements and their associated inaccuracies.

\subsection{A Single Measurement Model}

Polaroid sensors set aboard the vehicle have been widely used in mobile robotic applications by using Extended Kalman Filtering [12] or Bounded Error estimation [13]. Physical models of such sensors have been extensively studied in [10]. The sensor measures the time lag between the emission of an acoustic wave and its reception at the same place after its reflection. From time lag, the distance to the nearest reflective surface in the sonic ray direction can be computed. As any antenna, the sensor emits waves

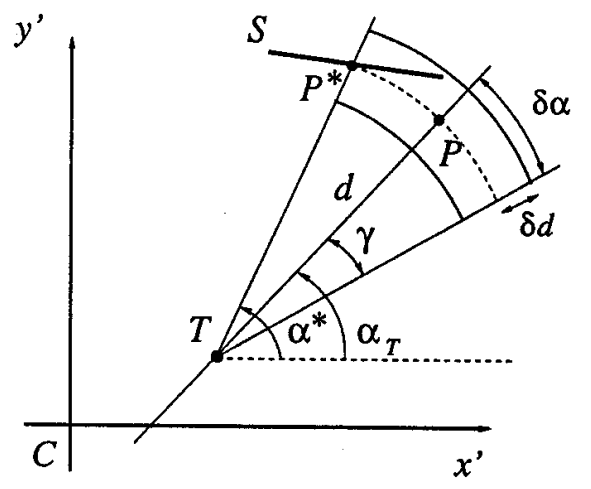

Figure 3: Sonar measurement model.

inside a cone whose axis is the sensor direction (see Figure 3). This implies that the sensor measures the distance between itself and the nearest object of the environment inside the emission cone. The fact that the object belongs to an a priori known map feature 
constitutes the measurement equation. In Figure 3, the sensor $T$ emits a wave reflected by a straight segment $S$. The detected surface element is around a segment point denoted by $P^{*}$ which is the nearest point to the sensor $T$ inside the cone. As the information given by the sensor $T$ is the distance $d$, one can state that the real direction $\alpha^{*}$ lies in $\left[\alpha_{T}-\gamma, \alpha_{T}+\gamma\right]$ where both $\alpha_{T}$ and $\gamma$ are known a priori. Taking a $2 \%$ distance measurement inaccuracy into account, the real distance $d^{*}$ is then inside the following interval:

$$
D_{1 m e a s}=[0.98 d, 1.02 d]
$$

\section{GUARANTEED ESTIMA- TION}

After stating the model of ultrasonic measurement inaccuracy, this section translates the localization problem into a set-inversion problem where one looks for the set of all configurations which are mapped into the admissible measurement domain by a simulation of the sensors behavior. Solving this set-inversion problem will be by means of interval analysis.

\subsection{Global Measurement Model}

Consider the set of $t$ sensors $T_{i}$ of the vehicle belt, defined in $\mathcal{M}$, and the set of $s$ map features $S_{j}$ defined in $\mathcal{W}$. The aperture angle of the emission cone of any sensor is assumed to be constant and equal to $2 \gamma$ (see Figure 3 ). The vehicle configuration is denoted by $\mathrm{q}=\left(x_{C}, y_{C}, \theta\right)^{T}$.

The following program defines a simulation function $f$ which maps $q \in \mathcal{C}$ in $d \in \Re^{t}$ :

$$
\left[\begin{array}{c}
x_{C} \\
y_{C} \\
\theta
\end{array}\right] \stackrel{\mathbf{f}}{\rightarrow}\left[\begin{array}{c}
d_{1} \\
d_{2} \\
\vdots \\
d_{t}
\end{array}\right]
$$

For all sensors $T_{i}$

Initialize $d_{i}=d_{\max }$

For all map features $S_{j}$

Compute $d_{i j}=$ distance of the nearest point of

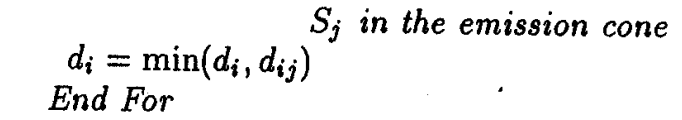

End For

The sensor configurations and the environment map are implicit data of the function definition. The set-inversion problem for the localization is then as follows: Knowing a feasible domain $\mathbf{D}$ for the measurement vector defined by (3) and the simulation function $\mathrm{f} 2$, what is the set $\mathcal{Q}$ which is mapped into $\mathbf{D}$ by $\mathbf{f}$ ?

$$
\mathrm{D}=\bigotimes_{i=1}^{t}\left[0.98 d_{i}, 1.02 d_{i}\right]
$$

\subsection{Set Inversion}

Consider a continuous computable function $f$ from $\Re^{n}$ into $\Re^{p}$. Let $\mathcal{Y}$ be a set in the image space $\Re^{p}$. A set inversion problem is that of finding the set $\mathcal{X}$ in $\Re^{n}$ so that:

$$
\mathcal{X}=\mathbf{f}^{-1}(\mathcal{Y})=\left\{\mathbf{x} \in \Re^{n} \quad \mid \mathbf{f}(\mathbf{x}) \in \mathcal{Y}\right\}
$$

The function $f^{-1}$ is the reciprocal image of the vectorial function $\mathbf{f}, \mathcal{Y}$ is the set to be inverted and $\mathcal{X}$ is the solution set of the set-inversion problem. According to results of topology theory, when $\mathcal{Y}$ is compact one can approximate the solution set by a union of boxes, as can be provided by interval analysis. Here, $f$ is the function defined by (2) and the set $\mathcal{Y}$ to be inverted is the measurement box defined by (3).

Interval analysis has been a very active field in scientific computation over the last 20 years [14]. The domain has matured enough for that the resulting methods can be implemented using software tools [17]. We focus on the use of the SIVIA algorithm [7] [9] to solve our localization problem. We will now recall the notions of interval analysis that will be used for the description of SIVIA, more details on interval analysis can be found in [14] and [6].

Consider an interval $X$ in $\Re$ :

$$
X=\left\{x \mid x^{-} \leq x \leq x^{+}\right\}=\left[x^{-}, x^{+}\right]
$$


which can be generalized in $\Re^{n}$ as the Cartesian product of $n$ scalar intervals (i.e. a box):

$$
\mathrm{X}=\bigotimes_{i=1}^{n}\left[x_{i}^{-}, x_{i}^{+}\right]
$$

Let $\Im \Re^{n}$ be the space of the boxes in $\Re^{n}$, and let $\mathbf{X}$ be a box in $\Im \Re^{n}$. The width of this box is defined by:

$$
w(\mathbf{X})=\max _{i=1, \ldots, n}\left(x_{i}^{+}-x_{i}^{-}\right)
$$

Consider a function $\mathbf{f}: \Re^{n} \longrightarrow \Re^{p}$. The image by $\mathbf{f}$ of any box $\mathbf{X}$ in $\Im \Re^{n}$ is a set $\mathbf{f}(\mathbf{X})$ in $\Re^{p}$, which usually cannot be computed exactly. With the help of the notion of inclusion function, however one can compute a box in $\Im \Re^{p}$ guaranteed to contain the set $\mathbf{f}(\mathbf{X})$. The function $[\mathbf{f}]: \Im \Re^{n} \longrightarrow \Im \Re^{p}$ is an inclusion function associated with $\mathbf{f}$ if it satisfies:

$$
\forall \mathbf{X} \in \Im_{\Re^{n}}, \mathbf{f}(\mathbf{X}) \subset[\mathbf{f}](\mathbf{X})
$$

Inclusion functions thus make it possible to compute boxes guaranteed to contain the image of $\mathrm{X}$ by $\mathbf{f}$. This approximation of $\mathbf{f}(\mathbf{X})$ by $[\mathbf{f}](\mathbf{X})$ may be very pessimistic but can be improved by considering smaller boxes provided that the inclusion function is inclusion monotonic:

$$
\forall \mathbf{X}_{1}, \mathbf{X}_{2} \in \Im \Re^{n}, \mathbf{X}_{1} \subset \mathbf{X}_{2} \Longrightarrow[\mathbf{f}]\left(\mathbf{X}_{1}\right) \subset[\mathbf{f}]\left(\mathbf{X}_{2}\right)
$$

Infinitely many inclusion functions can be derived for a given continuous function $\mathbf{f}$, and more efficient inclusion functions may be obtained by taking the intersection of the image of several inclusion functions. A simple way to obtain an inclusion function for any continuous function defined by an explicit formal expression (or program) is to replace all elementary operators such as,,$+- \times, /$ or functions such as cos, sin, exp by their interval counterparts. For example, if $A, B$ and $C$ are intervals of $\Im \Re$ so that $C=A+B$. Then the endpoints of $C$ are computed as:

$$
\left\{\begin{array}{l}
c^{-}=a^{-}+b^{-} \\
c^{+}=a^{+}+b^{+}
\end{array}\right.
$$

The result of these substitutions is called a natural interval extension of the function $\mathbf{f}$. Computing such natural interval extensions is readily performed by software libraries such as C-XSC [17].

Let [f] be an inclusion function for the function $f$ defined by 2 . Any box $\mathbf{X}$ such as $[\mathbf{f}](\mathbf{X}) \subset \mathcal{D}$ belongs to $\mathcal{Q}$. Any box $\mathbf{X}$ such as $[\mathbf{f}](\mathbf{X}) \cap \mathcal{D}=\emptyset$ has an empty intersection with $\mathcal{Q}$. These two tests make it possible to partition the initial search domain $\mathbf{X}(0)$ into three subpavings (union of boxes). $\mathcal{Q}_{\text {in }}$ consists of all boxes which have been proved to belong to $\mathcal{Q}$. $\mathcal{Q}_{\text {out }}$ consists of all boxes which have been proved to have an empty intersection with $\mathcal{Q}$. $\mathcal{Q}_{\text {ind }}$ consists of all boxes for which no conclusion could be drawn. Provided $\mathbf{X}(0)$ is large enough to contain $\mathcal{Q}$, these subpavings can be used to bracket the solution set $\mathcal{Q}$ between two subpavings:

$$
\mathcal{Q}_{\text {in }} \subset \mathcal{Q} \subset \mathcal{Q}_{\text {in }} \cup \mathcal{Q}_{\text {ind }}
$$

Any box in $\mathcal{Q}_{\text {ind }}$ can be split into subboxes to try to improve the precision of the bracketing by taking advantage of the inclusion monotonicity of the inclusion function. This splitting only takes place if the width of the indeterminate box is larger than some predefined tolerance $\epsilon$, so the algorithm is finite. Its convergence has been proved [8] under quite general conditions. The results obtained are global and guaranteed, even if the solution set is not connected, as illustrated in the next section. For the sake of brevity, the technical details of the algorithm are not described here. In Appendix A, an academic example is presented to illustrate the partition of the prior domain of interest into three subpavings by SIVIA. Hereafter, we present a simulation result which illustrates the characterization of the configuration domain.

\section{SIMULATION RESULTS}

The vehicle environment is a triangular room depicted by the Figure 4(a). Distance measurements in the form of ultrasonic range data are simulated according to the sensor model described in Subsection 2.3. In this experiment, the vehicle is placed in 
the configuration $\mathrm{q}^{*}=\left(-2 m, 3 m, 0^{\circ}\right)^{T}$, and four ultrasonic sensors are set in action (see Figure 4(a)). Thus, in this case four distance measurements are returned with a $2 \%$ distance relative precision and a $\pm 12^{\circ}$ direction absolute precision. The initial search domain is $\mathbf{X}(0)=[-10 m, 10 \mathrm{~m}] \times[-10 \mathrm{~m}, 10 \mathrm{~m}] \times$ $\left[-180^{\circ},+180^{\circ}\right]$ and the width of the smallest box to be explored is set to $\epsilon=0.04 \mathrm{~m}$. The resulting solution set is a non-connected set which consists of two connected component $\mathcal{Q}_{1}$ and $\mathcal{Q}_{2}$ which define two quite different types of configurations. To make interpretation easier, we choose to characterize $\mathcal{Q}_{1}$ and $\mathcal{Q}_{2}$ by boxes guaranteed to contain them, other set features like volume could be used as well. $\mathcal{Q}_{1}$ is inside the box $[-2.10 m,-1.87 m] \times[2.92 m, 3.11 \mathrm{~m}] \times$ $\left[-5.73^{\circ},+5.73^{\circ}\right]$ and $\mathcal{Q}_{2}$ is inside $[-3.09 m,-2.88 m] \times$ $[3.63 \mathrm{~m}, 3.81 \mathrm{~m}] \times\left[83.08^{\circ}, 91.67^{\circ}\right]$, (see Figures 4(b) and $4(\mathrm{c}))$. The first solution subset is the largest and contains the true vehicle configuration $\mathrm{q}^{*}$ whereas the second one reveals an ambiguity in data association. Indeed, $\mathcal{Q}_{1}$ corresponds to the matching hypothesis $h_{1}=\left\{\left(T_{1}, S_{1}\right)\left(T_{2}, S_{2}\right)\left(T_{3}, S_{2}\right)\left(T_{4}, S_{3}\right)\right\}$ (see Figure $5\left(\right.$ a)) while $\mathcal{Q}_{2}$ is related to the matching hypothesis $h_{2}=\left\{\left(T_{1}, S_{2}\right)\left(T_{2}, S_{3}\right)\left(T_{3}, S_{3}\right)\left(T_{4}, S_{1}\right)\right\}$ (see Figure 5(b)). So, these two sets correspond to two different placements of the vehicle which are consistent with very few (four) range measurements. Notice that each connected set is relatively narrow compared with common results obtained with sonars. Besides natural management of multiple hypothesis, the characterization of the admissible configuration domain is very accurate and guaranteed, as illustrated.

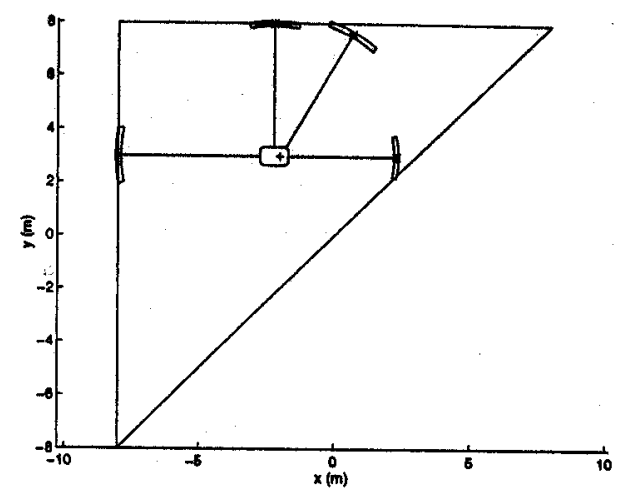

(a) Vehicle environment and simulated measurements.

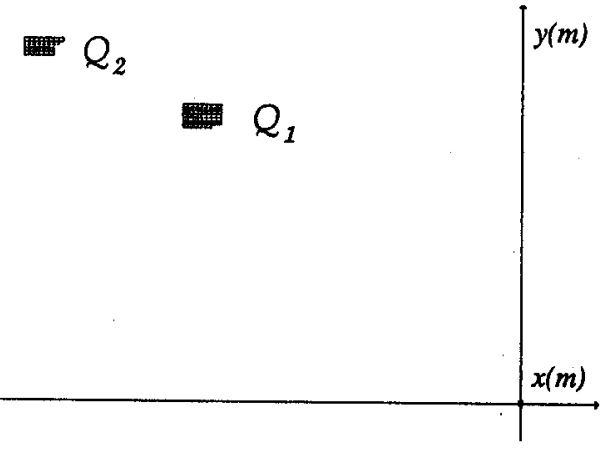

(b) Configuration domain in the plane $(x, y)$.

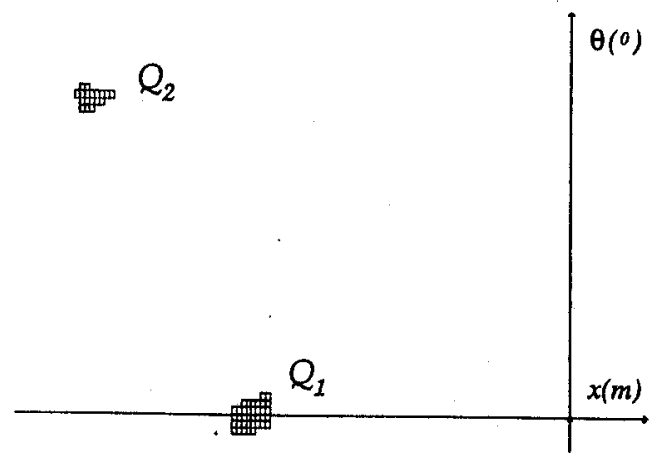

(c) Configuration domain in the plane $(x, \theta)$.

Figure 4: Configuration domain consistent with the measurements. To make the figure clearer, only boxes belonging to $\mathcal{Q}_{\text {in }} \cup \mathcal{Q}_{\text {ind }}$ are drawn. Other explored boxes are not represented. 


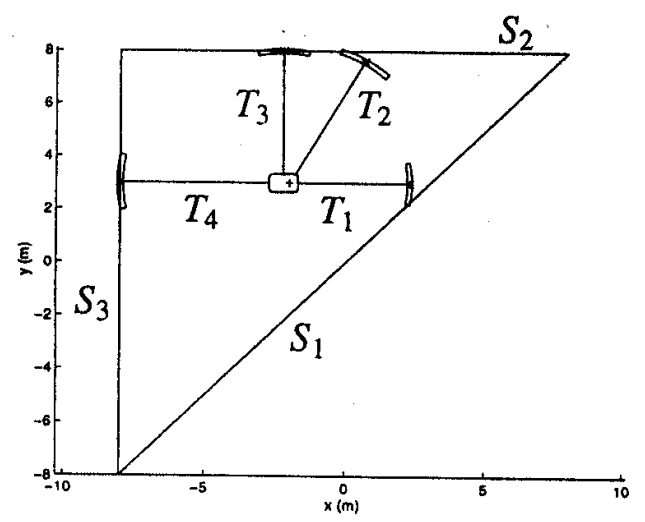

(a) First association hypothesis.

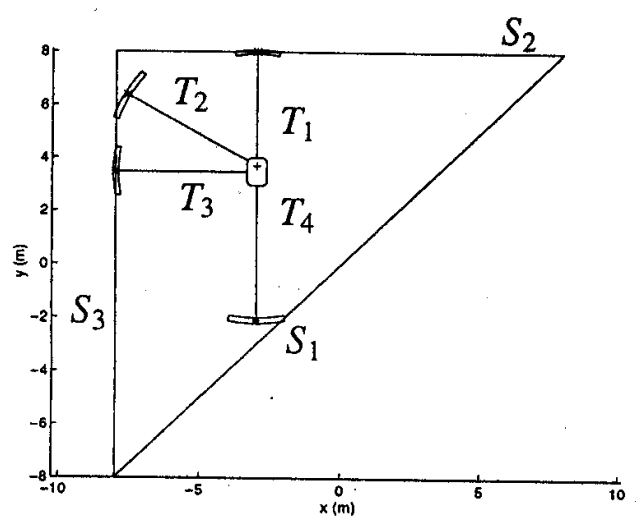

(b) Second association hypothesis.

Figure 5: Hypothesis of feature-measurement matching.

\section{CONCLUSION}

In this paper, a static vehicle localization methodology based upon set inversion via interval analysis has been presented. Vehicle localization problem has been defined as a set-inversion problem and solved by inverting the ultrasonic measurement model. The proposed methodology quickly eliminates large por- tions of the parameter space before focusing on the indeterminate region from which the solution set is deduced. The so obtained solution set is global, very accurate even using sonars which are individually inaccurate, and contains the real vehicle configuration if the error bounds on the measurements are underly optimistic. When the solution set is non-connected, the method generates multiple hypothesis which are derived from the connected components of the solution set and so reveals an ambiguity in the range data association. Thus, a set of hypothesis is defined, which can be further pruned by taking more range measurements into account. Contrary to classical statistical localization methods, the proposed approach does not need an initial localization and moreover there is no explicit matching step. The measurement-map matching application can be deduced as a side product for any connected part of the feasible domain.

\section{References}

[1] S. Atiya and G.D. Hager. Real-time vision-based robot localization. IEEE Trans. Robotics and Automation, 9(6):785-800, 1993.

[2] P. Bonnifait and G. Garcia. A multisensor localization algorithm for mobile robots and its realtime experimental validation. In Proc. IEEE Int. Conf. on Robotics and Automation, pages 13951400, Mineapolis, USA, 1996.

[3] A. Curran and J. Kyriakopoulos. Sensor-based self-localisation for wheeled mobile robots. $J$. of Robotic Systems, 12(3):163-176, 1995.

[4] E. Halbwachs and D. Meizel. Bounded-error estimation for mobile vehicle localization. In Proc. Int. Symp. on Robotics and Cybernetics, pages 1005-1010, Lille, France, July 1996.

[5] U.D Hanebeck and G. Schmidt. Set theoretic localization of fast mobile robots using an angle measurement technique. In Proc. IEEE Int. Conf. on Robotics and Automation, pages 13871394, Mineapolis, USA, 1996. 
[6] E. Hansen. Global Optimization using Interval Analysis. Marcel Dekker, New-York, 1992.

[7] L. Jaulin. Solution Globale et Garantie de Problèmes Ensemblistes. $\mathrm{PhD}$ thesis, Université de Paris Sud, Centre d'Orsay, 1994.

[8] L. Jaulin and E. Walter. Guaranted nonlinear parameter estimation from bounded-error data via interval analysis. Mathematics and Computers in Simulation, 35:123-127, 1993

[9] L. Jaulin and E. Walter. Set inversion via interval analysis for nonlinear bounded-error estimation. Automatica, 29(4):1053-1064, 1993.

[10] R. Kuc and M.W. Siegel. Physically based simulation model for acoustic sensor robot navigation. IEEE Trans. Pattern and Machine Intelligence, 9(6):766-778, 1987.

[11] J.J. Leonard and H.F. Durrant-Whyte. Mobile robot localization by tracking geometric beacons. IEEE Trar.s. Robotics and Automation, $7(3): 376-382,1991$.

[12] J.J. Leonard and H.F. Durrant-Whyte. Directed Sonar Sensing for Mobile Robot Navigation. Kluwer Academic Publishers, BostonLondon, 1992.

[13] H. Piet-Lahanier M. Milanese, J. Norton and E. Walter. Bounding Approaches to System Identification. Plenum, New-York, 1996.

[14] R.E. Moore. Methods and Applications of Interval Analysis. SIAM, Philadelphia, 1979.

[15] J.P. Norton. Bounded-error estimation: issue 1. Special Issue of Int. J. Adaptive Control and Signal Processing, 8:1-118, 1994.

[16] J.P. Norton. Bounded-error estimation: issue 2. Special Issue of Int. J. Adaptive Control and Signal Processing, 9:1-132, 1995.

[17] U. Kulisch R. Hammer, M. Hocks and D. Ratz. $\mathrm{C}++$ Toolbox for Verified Computing I - Basic Numerical Problems. Springer-Verlag, NewYork, 1995
[18] R.C. Smith and P. Cheeseman. On the representation and estimation of spatial uncertainty. Int. J. of Robotics Research, 5(4):56-68, 1986.

[19] E. Walter and H. Piet-Lahanier. Estimation of parameter bounds from bounded-error data: a survey. Mathematics and Computers in Simulation, 32(5):449-468, 1990.

\section{Appendix A: Basic example}

Consider the problem of characterizing the set of all points $(x, y)$ which satisfy the nonlinear inequalities system defined by the intersection between the unit disc (12) and a domain defined by the complementary of an ellipse centered in the origin (13). The initial search box is given by $\mathrm{X}(0)=[-2.5,2.5] \times[-2,2]$.

$$
\begin{aligned}
x^{2}+y^{2}-1 & \leq 0 \\
-2 x^{2}-y^{2}+1 & \leq 0
\end{aligned}
$$

Although the solution set is described by a set of nonlinear inequalities, we do not know for all that the elementary features of the set such as its shape and volume or whether it is empty or not. As illustrated by Figure 6, the solution set is characterized by SIVIA by means of two subpavings $\mathcal{Q}_{\text {in }}$ and $\mathcal{Q}_{\text {ind }}$ such as $\mathcal{Q}_{\text {in }} \subset \mathcal{Q} \subset \mathcal{Q}_{\text {in }} \cup \mathcal{Q}_{\text {ind }}$. The subpaving $\mathcal{Q}_{\text {out }}$ is thus defined as the complementary on the search box of $\mathcal{Q}_{\text {in }} \cup \mathcal{Q}_{\text {ind }}$.

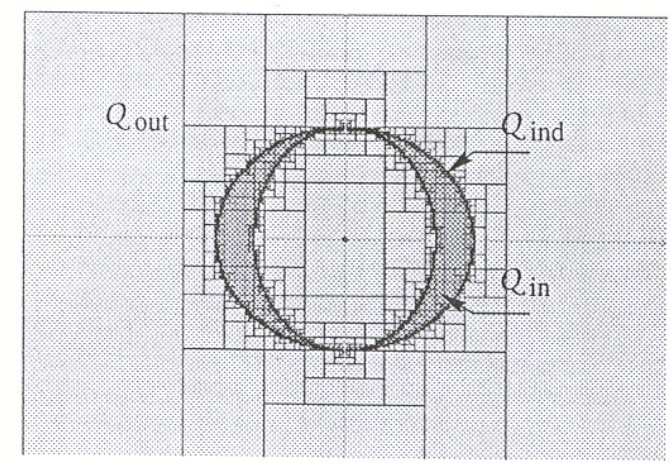

Figure 6: Solution set and resulting subpavings. 